Pacific Journal of Mathematics

A THEOREM ON THE ACTION OF ABELIAN UNITARY 


\title{
A THEOREM ON THE ACTION OF ABELIAN UNITARY GROUPS
}

\author{
William B. ARVESON
}

Given an abelian unitary group $G$ acting on the Hilbert space $\mathscr{H}$, let $\mathscr{A}$ be the $C^{*}$-algebra generated by $G$ and let $\sigma(\mathscr{A})$ denote the maximal ideal space of this algebra. There is a natural injection $\alpha$ of $\sigma(\mathscr{A})$ into the compact character group $\Gamma$ of the discrete group $G$. What conditions on $G$ will ensure that $\alpha$ be a topological homeomorphism of $\sigma(\mathscr{A})$ on $\Gamma$ ?

The action of $G$ is said to be nondegenerate if, for every finite subset $F$ of $G$, there exists a vector $\xi \neq 0$ in $\mathscr{H}$ such that $U \xi \perp V \xi$ for every pair $U, V$ of distinct elements of $F$. Theorem 1 contains the following answer to our question; in order that $\alpha$ map $\sigma(\mathscr{A})$ onto $\Gamma$, it is necessary and sufficient that the action of $G$ be nondegenerate.

To be more explicit, $\alpha$ is the mapping that merely restricts every complex homomorphism $\omega \in \sigma(\mathscr{A})$ to the group $G . \alpha$ is automatically continuous by definition of the topologies involved, and it is one-toone because a bounded linear functional on $\mathscr{A}$ is completely determined by its values on $G$, the latter being a fundamental set in $\mathscr{A}$. $\alpha$ will be a homeomorphism, therefore, provided only that every character in $\Gamma$ be the image of something in $\sigma(\mathscr{A})$.

Our interest in this problem arose out of a desire to characterize, in terms of action, when the spectrum of a unitary operator will fill out the unit circle. An appropriately translated version of Theorem 1 gives the following criterion: the spectrum of a unitary $U$ is the entire circle if, and only if, for every integer $n \geqq 1$ there exists a nonzero vector $\xi$ such that $\xi, U \xi, \cdots, U^{n} \xi$ are mutually orthogonal.

Versions of the sufficiency part of this problem have been considered before. Some time ago, Kodaira and Kakutani (6) showed essentially that $\alpha$ is onto $\Gamma$ when $G$ is the discrete unitary group determined by the left regular representation of a locally compact abelian group in its own $L_{2}$ space. Their proof involves the Plancherel theorem and is not available in this context. Recently, A. Ionescu-Tulcea (5) has shown that if $U$ is the unitary operator induced in $L_{2}$ of a $\sigma$-finite measure space by a nonperiodic invertible measure preserving transformation, then the spectrum of $U$ is the entire unit circle.

2. Examples. First, let us note that the definition of nondegener-

Received October 8, 1964. This paper contains a section from the author's doctoral dissertation submitted at the University of California at Los Angeles in September 1964. 
acy applies equally to unitary groups which are not necessarily abelian. Let $G$ be a unitary group on $\mathscr{H}$. Using the facts that $G$ is a group and that unitary operators preserve orthogonality, it is easily seen that nondegeneracy is equivalent to the following condition: for every finite subset $F$ of $G$ such that $I \notin F$, there exists a nonzero vector $\xi$ in $\mathscr{C}$ such that $\xi \perp U \xi$ for every $U$ in $F$.

The unitary group determined in $L_{2}$ by the left regular representation of a locally compact Hausdorff topological group is nondegenerate. This situation is really a special case of the following more general example from ergodic theory. As it is not our intention to enter a lengthy discussion of measure theoretic details for this example, we shall merely sketch results, all of which are known in one form or another. Let $X$ be a locally compact Hausdorff space and let $m$ be a regular Borel measure on the $\sigma$-algebra $\mathscr{B}$ of Borel sets in $X$ (4). By a measure preserving transformation (MPT) we mean a mapping $\sigma: X \rightarrow X$ such that, for every $B \in \mathscr{B}, \sigma^{-1}(B)=\{x \in X: \sigma(x) \in B\}$ belongs to $\mathscr{B}$ and $m\left(\sigma^{-1}(B)\right)=m(B)$. The set of all MPT's of $X$ form a semigroup $S$ with identity under the multiplication $(\sigma \tau) x=\sigma(\tau x), \sigma$, $\tau \in S, x \in X$. Let $G$ be a subgroup of $S$ whose identity is the identity of $S$. For $\sigma \in G$, define the operator $U_{\sigma}$ on $L_{2}(X, \mathscr{B}, m)$ by $\left(U_{\sigma} f\right)(x)=$ $f\left(\sigma^{-1} x\right), f \in L_{2}$. Then $\sigma \rightarrow U_{\sigma}$ is a unitary representation of $G$ on $L_{2}(X, \mathscr{B}, m)$. The group $G$ is said to be freely-acting if, for every $\sigma \in G$ different from the identity and every $B \in \mathscr{B}$ such that $m(B)>0$, there exists a Borel subset $A$ of $B$ such that $0<m(A)<\infty$ and $m\left(A \cap \sigma^{-1} A\right)=0$. This definition is esssentially von Neumann's and a discussion of it can be found in (3). If $G$ is a freely-acting group of MPT's, then by choosing nested subsets in the obvious way, we conclude that for every finite subset $\sigma_{1}, \cdots, \sigma_{n}$ of $G$ all different from the identity, and every Borel set $B$ such that $m(B)>0$, there exists a nonnull Borel subset $A$ of $B$ such that $m(A)<\infty$ and $m\left(A \cap \sigma_{k}^{-1} A\right)=0$ for $k=1,2, \cdots, n$. By considering the characteristic function of $A$ as an element of $L_{2}(X, \mathscr{B}, m)$, it follows, first, that the representation $\sigma \rightarrow U_{\sigma}$ is faithful $\left(\sigma \neq\right.$ identity $\rightarrow U_{\sigma} \neq I$ ) and, second, that the action of the image group $\left\{U_{\sigma}: \sigma \in G\right\}$ is nondegenerate.

Applying this to the first example, we need note merely that, with respect to left Haar measure on a locally compact group, the group of left translations constitutes a freely-acting group of MPT's.

As a second example, let $(X, \mathscr{S}, m)$ be a $\sigma$-finite measure space and let $\sigma$ be an invertible MPT on $X$ which is nonperiodic in the sense that, for every integer $n \geqq 1$, there exists a set $A=A_{n} \in \mathscr{S}$ such that $m\left(A_{\Delta} \sigma^{-n} A\right)>0, \Delta$ denoting the symmetric difference. Nonperiodicity is equivalent to the requirement that the unitary operator induced by $\sigma$ in $L_{2}(X, \mathscr{S}, m)$ generate an infinite cyclic group. Now 
it is not difficult to show that for every $n \geqq 1$, there exists a set $A \in \mathscr{S}$ such that $0<m(A)<\infty$ and $m\left(A \cap \sigma^{-k} A\right)=0$ for $k=1,2, \cdots, n$ (e.g., see [5]). Again, by considering the characteristic function of $A$ as an element of $L_{2}(X, \mathscr{S}, m)$, it follows that the infinite cyclic unitary group induced by $\sigma$ in $L_{2}$ is nondegenerate.

3. Action and spectrum. We turn now to the main result of this paper. The notation of $\S 1$ remains in force.

THEOREM 1. Let $G$ be an abelian unitary group on $\mathscr{H}$, generating the $C^{*}$-algebra $\mathscr{A}$. In order that the image of $\sigma(\mathscr{A})$ under the natural mapping $\alpha$ be all of $\Gamma$, it is necessary and sufficient that the action of $G$ be nondegenerate.

In this event, of course, $\alpha$ will be a homeomorphism. We begin the proof of sufficiency with an elementary lemma.

LEMMA 1. Let $G$ be any subset of the unitary group in an abelian $C^{*}$-algebra $\mathscr{A}$, and let $\gamma$ be any complex-valued function defined on $G$. In order that there exist an $\omega \in \sigma(\mathscr{A})$ whose restriction to $G$ is $\gamma$, it is necessary and sufficient that

$$
\inf _{\|\xi\|=1} \sum_{k=1}^{n}\left\|U_{k} \xi-\gamma\left(U_{k}\right) \xi\right\|=0
$$

for every finite subset $U_{1}, \cdots, U_{n}$ of $G$.

Proof. (Necessity) Let $\omega \in \sigma(\mathscr{A}), \quad U_{1}, \cdots, U_{n} \in G$. Clearly it suffices to show that

$$
\inf _{\|\xi\|=1} \sum_{k=1}^{n}\left\|U_{k} \xi-\omega\left(U_{k}\right) \xi\right\|^{2}=0
$$

Let

$$
A=\sum_{k=1}^{n}\left(U_{k}-\omega\left(U_{k}\right) I\right) *\left(U_{k}-\omega\left(U_{k}\right) I\right)
$$

Then $A$ is a positive operator in $\mathscr{A}$. If

$$
\inf _{\|\xi\|=1}(A \xi, \xi)=\varepsilon>0
$$

then $A-\varepsilon I \geqq 0$ which implies that $A$ is regular. But by construction, the image of $A$, under the Gelfand mapping, has a zero at $\omega \in \sigma(\mathscr{A})$, a contradiction.

For sufficiency, note first that $|\gamma(U)|=1$ for every $U \in G$. Indeed, 
if $\|\xi\|=1$ and $U \in G$, then

$$
\|U \xi-\gamma(U) \xi\| \geqq|\|U \xi\|-| \gamma(U)|\|\xi\||=|1-| \gamma(U)|| ;
$$

and by taking the infimum over $\|\xi\|=1$, we get $|1-| \gamma(U)||=0$.

For every $U \in G$, define

$$
K_{U}=\{\omega \in \sigma(\mathscr{A}): \omega(U)=\gamma(U)\} .
$$

We have to show that $\cap K_{U} \neq \phi$. Since each $K_{U}$ is a compact subset of $\sigma(\mathscr{A})$, it suffices to show that these sets have the finite intersection property. Fix $U_{1}, \cdots, U_{n} \in G$ and let

$$
A=n^{-1} \sum_{k=1}^{n} \bar{\gamma}\left(U_{k}\right) U_{k} \in \mathscr{A},
$$

the bar denoting complex conjugation. Then for every $\xi \in \mathscr{H}$,

$$
\begin{aligned}
\|A \xi-\xi\| & =\left\|n^{-1} \sum_{k=1}^{n}\left(\bar{\gamma}\left(U_{k}\right) U_{k} \xi-\xi\right)\right\| \\
& \leqq n^{-1} \sum_{k=1}^{n}\left\|\bar{\gamma}\left(U_{k}\right) U_{k} \xi-\xi\right\|=n^{-1} \sum_{k=1}^{n}\left\|U_{k} \xi-\gamma\left(U_{k}\right) \xi\right\| .
\end{aligned}
$$

So, by hypothesis, $\inf \|(A-I) \xi\|=0,\|\xi\|=1$, implying that $A-I$ is not regular. There exists, therefore, an element $\omega \in \sigma(\mathscr{A})$ such that

$$
1=\omega(I)=\omega(A)=n^{-1} \sum_{k=1}^{n} \bar{\gamma}\left(U_{k}\right) \omega\left(U_{k}\right) .
$$

Since each summand has unit modulus and 1 is an extreme point of the unit disc, we have $\bar{\gamma}\left(U_{k}\right) \omega(U)=1$ for $k=1,2, \cdots, n$. Therefore, $\omega \in K_{U_{1}} \cap \cdots \cap K_{U_{n}}$, completing the proof of Lemma 1 .

The author is indebted to Professor H. A. Dye for suggesting the following line of argument, thereby simplifying considerably the proof of sufficiency. The proof of Lemma 2 is based on an argument of Dixmier [2]. We shall write $|E|$ for the number of elements in the set $E$, and $E \backslash F$ for the set-theoretic difference consisting of those elements of $E$ not in $F$.

Lemma 2. Let $F$ be a finite subset of an abelian group $H$. Then for every $\varepsilon>0$ there exists a finite subset $S$ of $H$ such that

$$
|F S \backslash S| \leqq \varepsilon|S| \text {. }
$$

Proof. Say $F=\left\{x_{1}, x_{2}, \cdots, x_{n}\right\}$. For every $r \geqq 1$, let

$$
F_{r}=\left\{x_{1}^{r_{1}} x_{2}^{r_{2}} \cdots x_{n}^{r_{n}}: 1 \leqq r_{k} \leqq r\right\} .
$$

The sequence $F_{r}$ is increasing and $F F_{r} \subseteq F_{r+1}$. We claim $\left|F_{r+1}\right| \leqq$ 
$(1+\varepsilon)\left|F_{r}\right|$ for some $r$. Otherwise, $\left|F_{r+1}\right|>(1+\varepsilon)\left|F_{r}\right|$ for all $r \geqq 1$ and hence $\left|F_{r}\right|>(1+\varepsilon)^{r-1}\left|F_{1}\right|=(1+\varepsilon)^{r-1}$. This means that $(1+\varepsilon)^{r-1}<r^{n}$ for every $r \geqq 1$ since by construction $\left|F_{r}\right| \leqq r^{n}$, which is absurd.

Choose such an $r$, and let $S=F_{r}$. Then

$$
\begin{aligned}
|F S \backslash S| & \leqq\left|F_{r+1} \backslash F_{r}\right|=\left|F_{r+1}\right|-\left|F_{r}\right| \\
& \leqq(1+\varepsilon)\left|F_{r}\right|-\left|F_{r}\right|=\varepsilon|S|,
\end{aligned}
$$

proving Lemma 2.

Now let $F$ be a finite subset of the given group $G$, and let $\gamma \in \Gamma$. By Lemma 1, it suffices to show that for every $\varepsilon>0$ there exists $\xi \in \mathscr{H},\|\xi\|=1$, such that

$$
\max _{J \in F}\|U \xi-\gamma(U) \xi\|^{2}=\max _{U \in F}\|\bar{\gamma}(U) U \xi-\xi\|^{2} \leqq 2 \varepsilon .
$$

Let $F^{\prime}=\{\bar{\gamma}(U) U: U \in F\}$ and let $G^{\prime}$ be the group $\{\bar{\gamma}(U) U: U \in G\}$. It is clear that $G^{\prime}$ is a nondegenerate subgroup of the unitary group in $\mathscr{A}$. By Lemma 2, there exists a finite subset $S \subseteq G^{\prime}$ such that $\left|F^{\prime} S \backslash S\right| \leqq \varepsilon|S|$. By nondegeneracy, choose a nonzero $\zeta \in \mathscr{H}$ such that $V \zeta \perp W \zeta$ for all $W \neq V$ in $S \cup F^{\prime} S$.

Let $\xi=\sum_{V \in S} V \zeta$. Clearly $\|\xi\|^{2}=|S| \cdot\|\zeta\|^{2}>0$. If $W \in G^{\prime}$, then

$$
\begin{aligned}
W \xi-\xi & =\sum_{W S} V \zeta-\sum_{S} V \zeta \\
& =\sum_{W \backslash S} V \zeta-\sum_{S \backslash W S} V \zeta,
\end{aligned}
$$

since the summands cancel over $S \cap W S$. Now

$$
\begin{aligned}
|S \backslash W S| & =|S|-|S \cap W S|=|W S|-|S \cap W S| \\
& =|W S \backslash S| ;
\end{aligned}
$$

so that, if $W \in F^{\prime}$, then by orthogonality

$$
\begin{aligned}
\|W \xi-\xi\|^{2} & =(|W S \backslash S|+|S \backslash W S|)\|\zeta\|^{2} \\
& =2|W S \backslash S|\|\zeta\|^{2} \leqq 2\left|F^{\prime} S \backslash S\right|\|\zeta\|^{2} \\
& \leqq 2 \varepsilon|S|\|\zeta\|^{2}=2 \varepsilon\|\xi\|^{2} .
\end{aligned}
$$

The disired conclusion follows by normalizing $\xi$.

It remains to prove that the condition is necessary. Let $F$ be a finite subset of $G$ such that $I \notin F$. Assume first that $F$ contains both self-adjoint and non self-adjoint elements, the distinct self-adjoint unitaries being $U_{1}, \cdots, U_{m}$. For each of the remaining elements $V, V$ and $V^{-1}$ are distinct: we discard one of them from $F$ when (and only when) both are present. Let the distinct elements remaining be $V_{1}, \cdots, V_{n}$. Clearly the sets 


$$
\left\{U_{1}, \cdots, U_{m}\right\},\left\{V_{1}, \cdots, V_{n}\right\}, \quad \text { and } \quad\left\{V_{1}^{-1}, \cdots, V_{n}^{-1}\right\}
$$

are disjoint, and if

$$
F_{0}=\left\{U_{1}, \cdots, U_{m}, V_{1}, \cdots, V_{n}\right\},
$$

then $\xi \perp F \xi \Leftrightarrow \xi \perp F_{0} \xi$, for every $\xi \in \mathscr{H}$.

Now suppose $\xi \perp F_{0} \xi$ fails for every $\xi \neq 0$ in $\mathscr{H}$. Let $\mathcal{O}$ be the real vector space of bounded linear functionals $\rho$ on $\mathscr{A}$ which are self-adjoint in the sense that $\rho\left(T^{*}\right)=\bar{\rho}(T)$ for all $T$, and let $\Omega$ be the subset of $\mathcal{O}$ consisting of all canonical states $\omega_{\xi}(T)=(T \xi, \xi)\|\xi\|=1$. Observe that $\Omega$ is convex. For let $\xi, \eta \in \mathscr{H},\|\xi\|=\|\eta\|=1$, and take $\theta$ in the unit interval. Consider the linear functional

$$
\rho(T)=\theta \omega_{\xi}(T)+(1-\theta) \omega_{\eta}(T)
$$

defined on the weak closure $\mathscr{B}$ of $\mathscr{A}$. As $\rho$ is weakly continuous and $\mathscr{B}$ is an abelian von Neumann algebra, $\rho$ already has the form $\rho=\omega_{\zeta}$ for some $\zeta \in \mathscr{H}$ (see (1), p. 233). We have

$$
\|\zeta\|^{2}=\rho(I)=\theta\|\xi\|^{2}+(1-\theta)\|\eta\|^{2}=1
$$

and hence the restriction of $\rho$ to $\mathscr{A}$ is in $\Omega$.

Consider now the linear mapping

$$
\rho \in \mathcal{O} \rightarrow\left(\rho\left(U_{1}\right), \cdots, \rho\left(U_{m}\right), \rho\left(V_{1}\right), \cdots, \rho\left(V_{n}\right)\right)
$$

of $\mathcal{O}$ into the $m+2 n$-dimensional real vector space

$$
R^{m} \oplus C^{n}=\left\{\left(x_{1}, \cdots, x_{m}, z_{1}, \cdots, z_{n}\right): x_{i} \in R, z_{j} \in C\right\},
$$

where as usual $R$ and $C$ denote the real and complex number fields. The image $K$ of $\Omega$ is a convex subset of $R^{m} \oplus C^{n}$, and by our assumption on $F_{0}, K$ does not contain the origin. By a standard separation theorem, there exists a nontrivial real linear functional $f$ on $R^{m} \oplus C^{n}$ such that $f(K) \geqq 0$.

It is easily seen that $f$ has the form

$$
f\left(x_{1}, \cdots, x_{m}, z_{1}, \cdots, z_{n}\right)=\sum_{k=1}^{m} a_{k} x_{k}+\sum_{j=1}^{n} b_{j} z_{j}+\sum_{j=1}^{n} \bar{b}_{j} \bar{z}_{j}
$$

where $a_{k} \in R, b_{j} \in C$. Define the operator $T \in \mathscr{A}$ by

$$
T=\sum a_{k} U_{k}+\sum b_{j} V_{j}+\sum \bar{b}_{j} V_{j}^{-1} .
$$

For every $\xi \in \mathscr{H},\|\xi\|=1$, we have

$$
\begin{aligned}
\omega_{\xi}(T) & =\sum a_{k} \omega_{\xi}\left(U_{k}\right)+\sum b_{j} \omega_{\xi}\left(V_{j}\right)+\sum \bar{b}_{j} \bar{\omega}_{\xi}\left(V_{j}\right) \\
& =f\left(\omega_{\xi}\left(U_{1}\right), \cdots, \omega_{\xi}\left(U_{m}\right), \omega_{\xi}\left(V_{1}\right), \cdots, \omega_{\xi}\left(V_{n}\right)\right) \geqq 0 .
\end{aligned}
$$


Therefore, $T$ is positive. By hypothesis, we may identify $\sigma(\mathscr{A})$ with $\Gamma$ by virtue of the homeomorphism $\alpha$, the Gelfand mapping $A \in \mathscr{A} \rightarrow$ $\hat{A} \in C(\Gamma)^{1}$ taking $G$ isomorphically into the character group of $\Gamma$. The continuous function

$$
\widehat{T}=\sum a_{k} \hat{U}_{k}+\sum b_{j} \hat{V}_{j}+\sum \bar{b}_{j} \hat{V}_{j}^{-1}
$$

is nonnegative everywhere and its Haar integral is zero because the characters $\hat{U}_{k}, \hat{V}_{k}, \hat{V}_{k}^{-1}$ are all different from the function 1 . Hence $\hat{T}$ vanishes identically. But by construction the characters on the right are distinct and therefore linearly independent, so that

$$
a_{1}=\cdots=a_{m}=b_{1}=\cdots=b_{n}=0,
$$

contradicting our choice of $f$.

A parallel argument applies if the original set $F$ consists entirely of self-adjoint or non self-adjoint elements. One merely replaces $R^{m} \oplus C^{n}$ with $R^{m}$ or $C^{n}$ depending on which case occurs. This completes the proof of Theorem 1.

We conclude this discussion with a few remarks. If $G$ is any nondegenerate unitary group, $\mathscr{A}$ is the generated $C^{*}$-algebra, and $\Sigma(\mathscr{A})$ is the state space consisting of all positive linear functionals $\rho$ on $\mathscr{A}$ such that $\rho(I)=1$, then there exists an element $\varphi \in \Sigma(\mathscr{A})$ such that $\varphi(U)=0$ for every $U$ in $G$ different from the identity. Indeed, the sets

$$
K_{U}=\{\rho \in \Sigma(\mathscr{A}): \rho(U)=0\}
$$

are weak*-compact subsets of $\Sigma(\mathscr{A})$, and by definition of nondegeneracy, every finite intersection (for $U \neq I$ ) contains a canonical state. Thus

$$
\bigcap_{U \neq I} K_{U} \neq \varnothing
$$

Of course the state $\varphi$ is uniquely determined by this condition. If $G$ is abelian, then $\varphi$ may be identified with the Haar integral over $\Gamma=\sigma(\mathscr{A})$, and it is, therefore, faithful in the sense that $\phi\left(T^{*} T\right)=0$ implies $T=0$, for every $T \in \mathscr{A}$. In general, a simple continuity argument shows that $\varphi(S T)=\varphi(T S)$ for every $S, T \in \mathscr{A}$.

We intend to publish elsewhere a more complete discussion of the existence of a finite normal trace, with such properties, defined on the von Neumann algebra generated by a given discrete unitary group.

\footnotetext{
${ }^{1} C(\Gamma)$ denotes the algebra of all complex-valued continuous functions on $\Gamma$.
} 
The author gratefully acknowledges the many valuable suggestions and criticisms of Professor H. A. Dye during the course of that study.

\section{REFERENCES}

1. J. Dixmier, Les Algebres D'Operateurs Dans L'Espace Hilbertien, Gauthier-Villars, Paris, (1957).

2. L Les moyennes invariantes dans les semi-groupes et leur applications, Acta Sci. Math. Szeged., 12 (1950), 213-232.

3. H. A. Dye, On groups of measure preserving transformations I, Amer. J. Math. 81 (1959), 119-159.

4. E. Hewitt and K. Ross Abstract harmonic analysis, Academic Press, New York, (1963).

5. A. Ionescu-Tulcea, Tulane symposium on ergodic theory, Fred Wright, Editor, Academic Press, (1963).

6. K. Kodaira and S. Kakutani, Normed ring of a locally compact Abelian group, Proc. Imp. Acad. Tokyo, 19 (1943), 360-365.

U. S. Naval Ordnance Test Station, Pasadena, California

AND

HARVARD UNIVERSITY 


\section{PACIFIC JOURNAL OF MATHEMATICS}

\section{EDITORS}

\section{H. SAMELSON}

Stanford University

Stanford, California

\section{R. M. BLUMenthaL \\ University of Washington \\ Seattle, Washington 98105}

*J. DugundjI

University of Southern California Los Angeles, California 90007

\section{RICHARD ARENS}

University of California

Los Angeles, California 90024

\section{ASSOCIATE EDITORS}
E. F. BECKENBACH
B. H. NeUManN
F. WOLF
K. YosIDA

\section{SUPPORTING INSTITUTIONS}

\author{
UNIVERSITY OF BRITISH COLUMBIA \\ CALIFORNIA INSTITUTE OF TECHNOLOGY \\ UNIVERSITY OF CALIFORNIA \\ MONTANA STATE UNIVERSITY \\ UNIVERSITY OF NEVADA \\ NEW MEXICO STATE UNIVERSITY \\ OREGON STATE UNIVERSITY \\ UNIVERSITY OF OREGON \\ OSAKA UNIVERSITY \\ UNIVERSITY OF SOUTHERN CALIFORNIA
}

\author{
STANFORD UNIVERSITY \\ UNIVERSITY OF TOKYO \\ UNIVERSITY OF UTAH \\ WASHINGTON STATE UNIVERSITY \\ UNIVERSITY OF WASHINGTON \\ AMERICAN MATHEMATICAL SOCIETY \\ CHEVRON RESEARCH CORPORATION \\ TRW SYSTEMS \\ NAVAL ORDNANCE TEST STATION
}

Mathematical papers intended for publication in the Pacific Journal of Mathematics should be typewritten (double spaced). The first paragraph or two must be capable of being used separately as a synopsis of the entire paper. It should not contain references to the bibliography. Manuscripts may be sent to any one of the four editors. All other communications to the editors should be addressed to the managing editor, Richard Arens at the University of California, Los Angeles, California 90024.

50 reprints per author of each article are furnished free of charge; additional copies may be obtained at cost in multiples of 50 .

The Pacific Journal of Mathematics is published monthly. Effective with Volume 16 the price per volume (3 numbers) is $\$ 8.00$; single issues, $\$ 3.00$. Special price for current issues to individual faculty members of supporting institutions and to individual members of the American Mathematical Society: $\$ 4.00$ per volume; single issues $\$ 1.50$. Back numbers are available.

Subscriptions, orders for back numbers, and changes of address should be sent to Pacific Journal of Mathematics, 103 Highland Boulevard, Berkeley 8, California.

Printed at Kokusai Bunken Insatsusha (International Academic Printing Co., Ltd.), No. 6, 2-chome, Fujimi-cho, Chiyoda-ku, Tokyo, Japan.

PUBLISHED BY PACIFIC JOURNAL OF MATHEMATICS, A NON-PROFIT CORPORATION

The Supporting Institutions listed above contribute to the cost of publication of this Journal, but they are not owners or publishers and have no responsibility for its content or policies.

* Paul A. White, Acting Editor until J. Dugundji returns. 


\section{Pacific Journal of Mathematics}

\section{Vol. 16, No. 2 December, 1966}

Loren N. Argabright, Invariant means on topological semigroups ........ 193

William Arveson, A theorem on the action of abelian unitary groups ...... 205

John Spurgeon Bradley, Adjoint quasi-differential operators of Euler

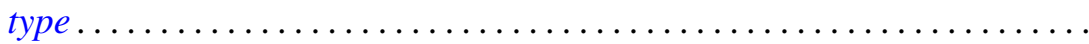

Don Deckard and Lincoln Kearney Durst, Unique factorization in power series rings and semigroups ........................... 239

Allen Devinatz, The deficiency index of ordinary self-adjoint differential operators..................................... 243

Robert E. Edwards, Operators commuting with translations ............ 259

Avner Friedman, Differentiability of solutions of ordinary differential equations in Hilbert space .............................. 267

Boris Garfinkel and Gregory Thomas McAllister, Jr., Singularities in a variational problem with an inequality ......................

Seymour Ginsburg and Edwin Spanier, Semigroups, Presburger formulas,

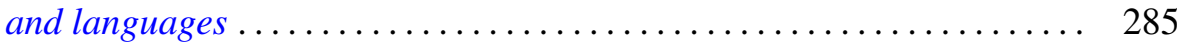

Burrell Washington Helton, Integral equations and product integrals . . . . . . 297

Edgar J. Howard, First and second category Abelian groups with the n-adic topology.........................................

Arthur H. Kruse and Paul William Liebnitz, Jr., An application of a family homotopy extension theorem to ANR spaces.

Albert Marden, I. Richards and Burton Rodin, On the regions bounded by homotopic curves

Willard Miller, Jr., A branching law for the symplectic groups ...

Marc Aristide Rieffel, A characterization of the group algebras of the finite

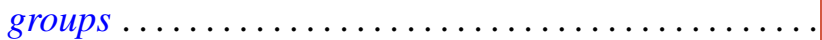

P. P. Saworotnow, On two-sided $H^{*}$-algebras

John Griggs Thompson, Factorizations of p-solvable groups ...

Shih-hsiung Tung, Harnack's inequalities on the classical Cartan

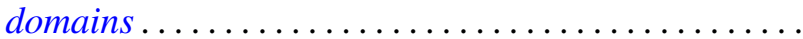

